cussed. These equations are in approximately the usual form, except for a change of variables. This change of variables is necessitated if tables are to be constructed to give all of the data desired for interior ballistics. The equations have been integrated numerically in the office of the Technical Staff of the Ordnance Department, and tables have been constructed which will shortly be published as a Government Document. The novelty of the undertaking consists in the introduction for the first time of new standard variables, which have a fundamental physical significance. The tables secured are independent of physical dimensions and appear to be the first to be published which correspond directly to the equations in their classical form. The results from the tables must be multiplied by external factors which involve the physical dimensions and depend upon the units chosen.

\title{
R. G. D. Richardson,
} Secretary.

\section{NOTE ON EQUAL CONTINUITY.}

BY DR. J. F. RITT.

(Read before the American Mathematical Society February 26, 1921.)

The notion of equal continuity, introduced by Ascoli, has acquired prominence through applications made of it by Hilbert, Montel and others.* A family of functions, defined on an interval, is equally continuous at a point if for every positive number there is an interval containing the point, in which the oscillation of each of the functions is less than the number.

Dealing with families of functions which are not equally continuous, we shall record here some properties of a function which will be called the saltus of the family.

Choosing a point on the interval of definition of the functions, let us suppose that there exists a number for which an interval can be found, containing the point, in which the oscillation of each of the functions is less than the number.

* For the literature on this question, see Montel, Sur les suites infinies de fonctions, ANNALES DE L'ECOLE NORMALE, 1907. 
The greatest lower bound of all such numbers will be called the saltus of the family at the point. If no such number exists, the saltus will be taken as $+\infty$. Of course, wherever the saltus vanishes, the family is equally continuous.

The saltus is an upper semi-continuous function on the interval considered. Consequently, if it vanishes nowhere in an interval, there is a sub-interval within that interval in which its lower bound exceeds zero.*

We shall apply the notion of the saltus of a family of functions to the case of a convergent sequence of continuous functions. Let the sequence of functions

$$
\varphi_{1}(x), \varphi_{2}(x), \cdots, \varphi_{n}(x), \cdots,
$$

each continuous in the interval $(a, b), \dagger$ converge throughout that interval. It is well known that wherever the sequence is equally continuous, the limit of the sequence is continuous.

Suppose that there be an interval $(\alpha, \beta)$ for no point of which the saltus of the sequence is zero. There must be an interval $\left(\alpha_{1}, \beta_{1}\right)$, within $(\alpha, \beta)$, for every point of which the saltus exceeds some positive number $\epsilon$. Let $\varphi_{i_{1}}(x)$ be any function of the above sequence, and $x_{0}$ any point of $\left(\alpha_{1}, \beta_{1}\right)$. In a small neighborhood of $x_{0}$, the oscillation of $\varphi_{i_{1}}(x)$ is very small. But for any such small neighborhood there is a function $\varphi_{i_{2}}(x)$, with $i_{2}>i_{1}$, whose oscillation in that neighborhood exceeds $\epsilon$. Hence there is an interval $\left(\alpha_{2}, \beta_{2}\right)$, interior to $\left(\alpha_{1}, \beta_{1}\right)$, in which $\varphi_{i_{2}}(x)$ differs from $\varphi_{i_{1}}(x)$ by more than $\epsilon / 3$. Continuing thus, we have a sequence of intervals

$$
\left(\alpha_{1}, \beta_{1}\right),\left(\alpha_{2}, \beta_{2}\right), \cdots,\left(\alpha_{n}, \beta_{n}\right), \cdots,
$$

each interior to the preceding one, and a sequence of functions

$$
\varphi_{i_{1}}(x), \varphi_{i_{2}}(x), \cdots, \varphi_{i_{n}}(x), \cdots,
$$

with $i_{n}>i_{n-1}$, such that

$$
\left|\varphi_{i_{n}}(x)-\varphi_{i_{n-1}}(x)\right|>\frac{\epsilon}{3}
$$

throughout $\left(\alpha_{n}, \beta_{n}\right)$. It is clear that the original sequence could not converge at the points common to the intervals $\left(\alpha_{n}, \beta_{n}\right)$.

* Hobson, Theory of Functions, p. 238.

$\dagger$ It does not matter whether the interval is taken as open or as closed. 
We find thus Baire's well known theorem to the effect that the limit of a sequence of continuous functions is at most point-wise discontinuous.

In connection with convergent sequences of continuous functions, the saltus function here considered can be related with the measure of non-uniform convergence introduced by Hobson and Osgood.* These two functions vanish at the same points, which fact shows, of course, that the above proof of Baire's theorem is not fundamentally distinct from that based on the measure of non-uniform convergence. There is no other relation of equality between the two functions.

Columbia University.

\section{A NEW METHOD IN DIOPHANTINE ANALYSIS.}

BY PROFESSOR L. E. DICKSON.

(Read before the American Mathematical Society March 26, 1921.)

1. Introduction and Summary. In the preceding number of this Bulletin (p. 312) I gave reasons why due caution should be observed toward the literature on the solution of homogeneous equations in integers. The valid knowledge concerning this subject is much less than has been usually admitted. The lack of general methods is even greater than in the subject of non-homogeneous equations. The chief aim of the present paper is to suggest such a method, based on the theory of ideals. The method is applicable in simple cases (\$§ 2-4) without introducing ideals.

For the sake of brevity we shall restrict attention to the problem of finding all integral solutions of the equation

$$
x_{1}^{2}+a x_{2}^{2}+b x_{3}^{2}=x_{4}^{2},
$$

an equation admitted $\dagger$ to be difficult of treatment by any known methods, and previously solved completely in integers only in the single case $a=b=1$.

Let us write

$$
x_{4}-x_{1}=z, \quad x_{4}+x_{1}=w .
$$

Then from the integral solutions of $a x^{2}+b y^{2}=z w$ we must

* Hobson, loc. cit., p. 484.

† Carmichael, Diophantine Analysis, 1915, p. 38. 